\title{
Gestão de materiais médico-hospitalares numa rede hospitalar pública utilizando matriz ABC/XYZ
}

\section{Medical-hospital materials management in a public hospital network using $\mathrm{ABC} / \mathrm{XYZ}$ matrix}

\author{
Jaqueline Daniela de Oliveira Fonseca \\ Centro Federal de Educação Tecnológica de Minas Gerais - CEFET-MG - Brasil \\ jaquelinedaniella@yahoo.com.br \\ ORCID: https://orcid.org/0000-0001-5784-9919 \\ Elisangela Martins de Sá \\ Centro Federal de Educação Tecnológica de Minas Gerais - CEFET-MG - Brasil \\ elisangelamartinss@gmail.com \\ ORCID: https://orcid.org/0000-0002-7801-0087
}

Fabrício Molica de Mendonça

Universidade Federal de São João Del Rei - UFSJ- Brasil

fabriciomolica@yahoo.com.br

ORCID: https://orcid.org/0000-0002-3175-1064

Paulo Fernandes Sanches Junior

Centro Federal de Educação Tecnológica de Minas Gerais CEFET-MG- Brasil

sanches@cefetmg.br

ORCID: https://orcid.org/0000-0002-1786-867X

Recebido: 24 Março 2021
Revisado: 03 Maio 2021
Aceito: 04 Agosto 2021

\section{Resumo}

Objetivo: o objetivo desta pesquisa é propor políticas de aquisição de estoque de materiais médicohospitalares em uma organização pública, que considerem variedade e quantidade adequadas à demanda, ajustando-se aos recursos financeiros e às legislações relacionadas, utilizando matriz ABC/ XYZ. Metodologia: Trata-se de estudo quantitativo desenvolvido na Fundação Hospitalar do Estado de Minas Gerais, maior rede de hospitais públicos da América Latina, utilizando pesquisa documental e métodos estatísticos, que estabeleceu estoques de segurança, ponto de pedido, lotes de compras e políticas de gestão de estoques. Resultados: A classificação é relevante para a gestão, diante da variabilidade dos itens, e demonstra que outros critérios, além do financeiro, como a variação da demanda, devem ser considerados na gestão de materiais na saúde pública. Contribuições: 0 estudo contribui no planejamento das compras, ao classificar o inventário de acordo com seu custo e rotatividade e incentivar o uso das atas de registro de preços, ocasionando economia de recursos financeiros ao diminuir estoque e perdas, apresentando projeção inicial de redução de $\mathrm{R} \$$ 3.900.006,43 - 8,6\% do gasto anual. O trabalho contribui para o campo teórico ao oferecer um modelo de aquisição e gestão de materiais que inclui também a variação da demanda de materiais e que atende às particularidades da saúde pública, ao considerar a imprescindibilidade e a diversidade dos insumos e as normativas legais aplicáveis.

Palavras-chave: Matriz ABC/XYZ; gestão de estoques; material médico-hospitalar; compras públicas

\section{Abstract}

Purpose: The objective of this research is to propose inventory policies for the acquisition of medicalhospital materials in a public organization, which consider demand-adequate variety and quantity, adjusting to both financial resources and related legislation, using ABC/XYZ matrix. Methodology: This is a quantitative study carried out at the Hospital Foundation of the State of Minas Gerais, the largest network of public hospitals in Latin America, applying documentary research and statistical methods, which established safety stocks, order points, purchase batches and inventory management policies. Results: The classification is relevant for management, given the variability of the items, as well as it shows that other criteria than financial, such as demand variation, must be considered in the management of materials in public health. Contribution: This study contributes to the planning of purchases, by classifying the inventory according to its cost and turnover, encouraging the use of 
pricing records, resulting in savings in financial resources by reducing both inventory and losses, which presents an initial decrease projection of $\mathrm{R} \$ 3.900 .006,43-8.6 \%$ of annual spending. The work contributes to the theoretical field by offering a material acquisition and management model that also includes the variation in the demand for materials and that meets the particularities of public health, when considering the indispensability and diversity of inputs and the applicable legal regulations.

Keywords: $\mathrm{ABC} / \mathrm{XYZ}$ matrix; inventory management; medical hospital material; public procurements

\section{Introdução}

No mundo pós-moderno, existe uma pressão do ambiente externo, em face das alterações legais, políticas e tecnológicas, bem como das exigências dos clientes, por menores preços e alto nível de serviço. Assim, a Administração Pública também está inserida nesse cenário de exigências, destacando-se as restrições de orçamento que pode sofrer (Almeida, Tavares, Melo, Nunes \& Negrão, 2016). Nesse contexto, algumas das práticas que merecem destaque são a aquisição de bens e o gerenciamento de estoque. A eficiência é um dos princípios básicos da Administração Pública (Campos \& Maria, 2015). Porém, alguns estudos (Araújo, Araújo \& Musetti, 2012; Gonçalves, Gonçalves, Tavares, Melo \& Nunes, 2015; Tridapalli, Fernandes \& Machado, 2011) apontam que a Administração Pública brasileira ainda não emprega metodologias adequadas para planejar suas aquisições, destacando algumas características arraigadas do setor, a saber: exigências legais, influência de política externa, aversão à inovação e descontinuidade de governos, que se tornam obstáculos para a profissionalização dessa tarefa. Na saúde pública, que é estudada neste trabalho, essa situação também é observada. Araújo et al. (2012) e Gonçalves et al. (2015) discorrem que a gestão de suprimentos na saúde pública está defasada em relação a outros ramos, por ser tratada apenas como custo e não como um fator estratégico. Embora a insuficiência de recursos financeiros seja um impasse para a melhoria da saúde pública, sendo recorrentemente indicada como o maior problema, o mau uso de materiais e equipamentos, o despreparo dos gestores e o planejamento precário também são empecilhos para o avanço no setor (Raimundo, Dias \& Guerra, 2015).

Os inventários hospitalares são variados, sendo, assim, um dificultador para se organizarem políticas de reposição de estoques (Fuccia, Matsufugi, Martins, Sforsin, \& Pinto, 2017). Os insumos nesse tipo de serviço são indispensáveis, diante da incerteza sobre em que momento será necessário cada material, e a ausência de algum item pode acarretar perdas irreparáveis para os pacientes e para as organizações. 0 custo também é um fator determinante, visto que, no Brasil, os gastos com insumos e serviços podem atingir 50\% das despesas de um hospital e, segundo o IBGE, cerca de $9 \%$ do Produto Interno Bruto (PIB) é destinado à aquisição de materiais e serviços para a área da saúde (Araújo et al., 2012). Destaca-se, ainda, que a aquisição de materiais e serviços no setor público brasileiro é determinada por regramentos legais, não se estabelecendo parcerias, como ocorre no meio privado. As compras públicas estão sujeitas à licitação, que nos últimos anos permite a utilização de pregão eletrônico e sistema de registro de preços.

Almeida et al. (2016) consideram que o melhoramento da administração de suprimentos na área pública brasileira necessita de uma gestão logística que atenda às suas peculiaridades. Entretanto, há uma lacuna desse tipo de pesquisa, o que dificulta a utilização de algum modelo. Araújo et al. (2012) apontam que os hospitais, sobretudo os públicos, são deficientes em gestão administrativa e não têm a visão de que ela precisa ser melhorada. Pesquisas utilizam ou apontam o critério financeiro como principal ou único fator para planejamento de compras no campo da saúde, em que os itens com maior valor recebem maior atenção, ao passo que os de menor valor são adquiridos com menor periodicidade, estando, por tal motivo, sujeitos à falta, comprometendo atividades mais complexas, desconsiderando a variação de demanda (Alvear \& Mello, 2016; Duarte et al., 2015; Figueiredo \& Aquino, 2016; Günergören \& Dağdeviren, 2017; Santos, Fank \& Varela, 2012). Além de faltas, pode haver, ainda, excessos de estoques, que levam a perdas por não utilização antes da sua validade ou deformação, quando o nível de estoque não é dimensionado adequadamente (Alvear \& Mello, 2016). Portanto, a gestão dos estoques é feita de maneira desintegrada, não orientada à demanda e sem cooperação com fornecedores, por impedimentos legais (Santos, Scheidegger, Favaretto \& Lima, 2014). Assim, devem-se buscar aquisições mais otimizadas, que supram as necessidades dos hospitais públicos, incorrendo em menor custo.

Dessa forma, é necessário que o Poder Público adote práticas que permitam melhor planejamento e controle, ou as importe (e adeque) do setor privado, buscando manter um nível de serviço mínimo à sociedade, sem demasiados custos, sendo recomendável estudar a realidade da organização para adequar procedimentos e modelos (Santos et al., 2014). Melo et al. (2016) e Raimundo et al. (2015) lembram da complexidade inerente a esse tipo de organização, tendo em vista os diferentes níveis de assistência prestados, e recomendam planejamento e controle adequado das aquisições e dos estoques a fim de se evitarem as compras emergenciais, que apresentam maiores 
custos para o Poder Público. Diante desse contexto, é necessária uma gestão de materiais que permita avaliar todos os itens e adequar os estoques a sua demanda e rotatividade, a fim de se evitarem falta e excessos e de se buscarem menores custos.

A organização estudada neste trabalho é composta por uma rede de hospitais públicos, com diversos níveis de complexidade, levando a necessidades distintas de itens. 0 problema de pesquisa que se pretende responder com este trabalho é: como a classificação dos materiais, utilizando os critérios financeiro e de variação da demanda, pode auxiliar na aquisição e no controle de suprimentos médico-hospitalares numa organização pública, considerando as peculiaridades da área? Assim, o objetivo geral deste artigo é propor políticas de gestão de materiais médico-hospitalares, que considerem as particularidades da área, como restrições de recursos financeiros, imprescindibilidade de materiais para as atividades de assistência à saúde e exigências das legislações referentes às compras públicas, por meio da análise $\mathrm{ABC}$ /XYZ. Os objetivos específicos são: (a) aplicar técnicas de gestão de estoque, para elaborar as classificações ABC e XYZ e a matriz ABC/XYZ; e (b) aplicar políticas de estoque para estimar estoque de segurança, ponto de pedido e lote de compra. A partir disso, pretende-se que este trabalho auxilie os gestores no processo decisório a respeito da aquisição e do controle de suprimentos médico-hospitalares, permitindo adequação dos inventários à demanda, economia de recursos financeiros e maior disponibilidade dos materiais para manutenção da assistência prestada. Espera-se, ainda, que esta pesquisa possa contribuir para o arcabouço teórico sobre o tema, ao oferecer uma metodologia para melhorar o planejamento e a gestão dos estoques de materiais médico-hospitalares, objetivando evitar a falta desses itens no estoque, bem como seu excesso e obsolescência, e atender com maior rapidez aos pedidos.

\section{Contexto da realidade investigada}

A Fundação Hospitalar do Estado de Minas Gerais (FHEMIG), que é retratada neste trabalho, é composta por uma rede de vinte hospitais, que oferecem tratamentos de diferentes especialidades médicas e com diversos níveis de complexidade, e pelo MG Transplantes, o que gera necessidades de diferentes materiais. Essas unidades estão localizadas em nove cidades do estado de Minas Gerais, incluindo a capital, a região metropolitana e o interior, sendo que algumas atendem mais de um serviço, e um prédio que abriga exclusivamente funções administrativas, localizado em Belo Horizonte. 0 orçamento geral empenhado em 2019 destinado aos materiais médico-hospitalares foi de, aproximadamente, $\mathrm{R} \$ 35.915 .000,00$ (Minas Gerais, 2021). É a maior rede de hospitais públicos da América Latina, prestando atendimento totalmente pelo Sistema Único de Saúde, sendo vinculada à Secretaria de Estado de Saúde de Minas Gerais (Alemão, Gonçalves \& Drumond, 2013).

Por se tratar de entidade pública, está obrigada a fazer licitação para aquisição de materiais e serviços. A Fundação utiliza o pregão eletrônico e as atas de registro de preços, regulamentadas, atualmente, pelo Decreto no 7.892 de 23 de janeiro de 2013, que apresenta as regras para contratação por meio do sistema de registro de preços (SRP), definido como "conjunto de procedimentos para registro formal de preços relativos à prestação de serviços e aquisição de bens, para contratações futuras", que é fundamentado na ata de registro de preços (ARP), a qual significa "documento vinculativo, obrigacional, com característica de compromisso para futura contratação, em que se registram os preços, fornecedores, órgãos participantes e condições a serem praticadas, [...]" (Decreto $\mathrm{n}$ - 7.982, 2013). A FHEMIG busca sempre ter ARP vigente para todos os materiais. Quando um registro está próximo de vencer, o setor responsável inicia processo para realizar o pregão para a assinatura de uma nova ata. Os materiais médico-hospitalares são adquiridos por meio de cerca de 17 pregões, que geram as atas, e os processos duram até 90 dias entre o início e a assinatura da ata.

\section{Diagnóstico da situação problema}

Segundo Mahagaonkar e Kelkar (2017), entre os objetivos primários da administração de materiais estão: planejamento efetivo do material; baixo custo das compras; controle de estoque eficiente; boas relações com fornecedores; e sistemas de informação adequados. Melo et al. (2016) discorrem que o estoque representa qualquer bem armazenado, e existe para conciliar as diferenças entre abastecimento e demanda. A classificação dos itens é útil, pois permite melhor controle e oferece apoio à decisão sobre estoque (Ferreira, Luz \& Amorim, 2016). Almeida et al. (2016) lembram que a gestão de estoques deve ter por objetivo, também, evitar os desperdícios, considerando o custo financeiro envolvido. As organizações públicas têm, adicionalmente, a obrigação de prestar contas de seus estoques, por exigências legais quanto à transparência do uso dos recursos financeiros e de seu patrimônio. Assim, esse fato tem levado alguns órgãos a adotar técnicas para otimizar a administração dos estoques (Souza, Cunha \& Leite, 2016). Para Souza e Rosa (2015), a administração de materiais hospitalares é mais complexa, devido às características dos insumos - prazo de validade, armazenagem, rastreabilidade. Hafnika, Farmaciawaty, Adhiutam \& Basri (2016) também concordam 
que a gestão de materiais em hospitais é mais complexa que na área industrial, considerando que há itens que devem ser fornecidos com alto nível de serviço e outros que devem receber atenção a fim de se evitar a perda. Meaulo e Pensutti (2011) completam que uma administração de materiais adequada deve buscar, entre outras atividades, a identificação de itens, o controle de estoques, o cálculo de médias de consumo, o levantamento dos principais custos envolvidos, a aplicação de algumas técnicas, como Curva ABC, Curva XYZ e Lote Econômico de Compra, e o constante acompanhamento dos índices de estoque (giro, excesso e lead time). Dentre as técnicas empregadas para a gestão de estoques, destacam-se: Curva $\mathrm{ABC}$, Curva XYZ, ponto de pedido e estoque de segurança.

A grande diversidade de itens que podem existir no estoque torna necessária sua classificação, para melhor controle e tomada de decisão. Assim, a técnica da Curva ABC - ou Curva de Pareto - busca classificar os insumos mediante o valor financeiro, a fim de apontar quais os materiais são mais importantes. A classificação A representa os recursos com maior valor agregado, embora com um número reduzido de bens. $O$ grupo $B$ é formado por elementos medianos e o $C$ abrange uma quantidade maior de materiais, com baixo valor financeiro. Assim, constrói-se uma hierarquia dentro do estoque (Duarte et al., 2015; Figueiredo \& Aquino, 2016; Gonçalves et al., 2015; Santos et al., 2012). Nesse sentido, Santana e Piper (2017) afirmam que essa técnica permite que os itens com maior valor sejam revisados com mais frequência que aqueles com custo menor, que, por sua vez, devem ser adquiridos ou produzidos com menor periodicidade, a fim de não sobrecarregar os setores envolvidos. A classificação $A B C$ é uma das técnicas mais utilizadas para controle de estoques na área da saúde (Günergören \& Dağdeviren, 2017).

Entretanto, a utilização da classificação $\mathrm{ABC}$, apenas, pode ignorar outros aspectos relevantes para o controle do estoque, levando ao menosprezo excessivo dos itens do grupo C, que podem ser importantes para algum subprocesso. 0 gestor não deve ignorar os bens de menor custo financeiro. A curva $\mathrm{ABC}$, embora importante para o controle dos estoques, é limitada pelo fato de considerar apenas o valor financeiro dos materiais (Gonçalves et al., 2015; Mahagaonkar \& Kelkar, 2017). Clevert, Stickel, Jung, Reiser \& Rupp (2007) alertam que a classificação ABC não é suficiente para otimizar o inventário, pois não avalia as flutuações que a demanda pode sofrer, e defendem que observar o estoque por um período maior possibilita identificar os produtos com consumo relativamente mais constante e aqueles com alguma variação ou demanda muito irregular. Assim, a possibilidade de predizer padrões de consumo pode ser avaliada por meio da curva XYZ. Stojanović e Regodić (2017) esclarecem que essa classificação busca adequar o estoque ao consumo. A classificação XYZ pode ser determinada com a utilização de dados estatísticos anuais ou elaborada por compradores ou médicos, e pode considerar informações acerca da variação dos preços (Clevert et al., 2007).

Dessa forma, a presente pesquisa baseou-se em metodologia quantitativa, buscando dados numéricos para medir acontecimentos e/ou delimitá-los em categorias mensuráveis, observando o relacionamento entre as variáveis ao longo do tempo. Para obter tais dados, foi realizada uma pesquisa documental, que, conforme defendem Marconi e Lakatos (2010), é a coleta de dados exclusivamente de documentos que constituem fontes primárias. Por meio da análise dos relatórios emitidos pelos sistemas de informação da organização estudada, obtiveram-se dados que demonstram valores e quantidades dos itens adquiridos, para a construção da Curva ABC, e variação do consumo mensal, para a Análise XYZ. Tais dados permitiram estabelecer, posteriormente, o estoque de segurança e o ponto do pedido. Finalmente, foi realizada uma simulação de redução de gastos utilizando-se as políticas propostas.

Segundo Mahagaonkar e Kelkar (2017), para construir a curva ABC, é necessário: (a) determinar o custo unitário de cada item e suas respectivas quantidades; (b) multiplicar o custo unitário pelo quantitativo, obtendo o custo por item; (c) listar os materiais, em ordem decrescente de custo; (d) somar os valores apurados, item a item, informando as frequências acumuladas; e (e) fazer um gráfico que relacione a porcentagem de itens com a porcentagem do custo total e delimitar as áreas correspondentes aos grupos A, B e C. Após esses procedimentos, devem-se considerar, na classe A, os itens que abrangem $80 \%$ do custo acumulado; na classe B, aqueles que comprometem $15 \%$ dos recursos financeiros; os 5\% restantes referem-se à classe C (Santana \& Piper, 2017).

Clevert et al. (2007) alertam que a Curva ABC não é suficiente para otimizar o inventário, pois não avalia as flutuações da demanda. Para Besta, Janovská e Bumbová (2015), o segundo fator crucial na administração de estoques é a variação do consumo. Assim, a curva XYZ foca a regularidade do consumo, sendo utilizados alguns indicadores estatísticos, entre eles, o coeficiente de variação. A classificação dos itens nas três categorias da Curva XYZ será baseada no trabalho de Eleftheria (2017), em que o coeficiente de variação até 33\% representa a classe X; de 33,1\% a 67\% indica o grupo Y; e acima de $67 \%$ enquadra-se na categoria $\mathrm{Z}$. A fórmula a seguir ilustra o cálculo do coeficiente de variação, conforme Besta et al. (2015). 0 resultado é multiplicado por 100 para expressar os valores em porcentagem. 
$V=\frac{\sigma}{c} x 100$

em que:

$V=$ coeficiente de variação;

$\sigma=$ desvio padrão;

$C=$ média do consumo.

Inicialmente, foram elaboradas as duas curvas separadamente; depois, os materiais foram alocados nas categorias criadas pela associação das técnicas. Assim, são formadas nove subcategorias: AX, AY, AZ, BX, BY, BZ, CX, CY e CZ. Na visão de Stojanović e Regodić (2017), as classificações AX, BX e AY devem adotar políticas de estoques mais baixos, devido ao custo mais elevado, enquanto os itens das categorias BZ, CY e CZ podem ser adquiridos com menos frequência, considerando demanda mais ocasional e menor valor, o que acarreta maior inventário. 0 uso simultâneo das técnicas auxilia na determinação de um nível ótimo de estoque, possibilitando a definição de práticas diferentes para cada grupo de itens. Assim, propõe-se que os itens AX, BX, BY, CX e CY tenham uma política de revisão contínua, enquanto AY, AZ, BZ e CZ tenham uma política de revisão periódica. As Figuras 1 e 2 ilustram as subcategorias formadas pelo emprego das duas técnicas e as políticas de estoque recomendadas, respectivamente:

Figura 1 - Análises ABC/XYZ combinadas

\begin{tabular}{|c|c|c|c|}
\hline Previsão $\backslash$ Valor & A (alto) & B (médio) & C (baixo) \\
\hline $\mathbf{X ~ ( a l t o ) ~}$ & $\mathrm{AX}$ & $\mathrm{BX}$ & $\mathrm{CX}$ \\
\hline Y (médio) & $\mathrm{AY}$ & $\mathrm{BY}$ & $\mathrm{CY}$ \\
\hline $\mathbf{Z}$ (baixo) & $\mathrm{AZ}$ & $\mathrm{BZ}$ & $\mathrm{CZ}$ \\
\hline
\end{tabular}

Fonte: Adaptado de Clevert et al. (2007)

Figura 2 - Controles de reposição de estoques

\begin{tabular}{|c|c|c|c|}
\hline Classificação & Itens A & Itens B & Itens C \\
\hline Itens X & $\begin{array}{c}\text { Demanda contínua } \\
\text { Baixo estoque }\end{array}$ & $\begin{array}{c}\text { Demanda contínua } \\
\text { Baixo estoque }\end{array}$ & $\begin{array}{c}\text { Demanda contínua } \\
\text { Baixo estoque }\end{array}$ \\
\hline Itens Y & $\begin{array}{c}\text { Demanda contínua } \\
\text { Baixo estoque }\end{array}$ & $\begin{array}{c}\text { Demanda flutuante } \\
\text { Médio estoque }\end{array}$ & $\begin{array}{c}\text { Demanda flutuante } \\
\text { Alto estoque }\end{array}$ \\
\hline Itens Z & Demanda flutuante & $\begin{array}{c}\text { Demanda flutuante } \\
\text { Médio estoque }\end{array}$ & $\begin{array}{c}\text { Demanda flutuante } \\
\text { Alto estoque }\end{array}$ \\
\hline
\end{tabular}

Fonte: Adaptado de Stojanović e Regodić (2017)

Assim, Póvoa e Chaves (2014) diferenciam as políticas de revisão contínua das políticas de revisão periódica. A revisão contínua permite que as práticas de reabastecimento sejam revistas a qualquer época, exigindo um controle constante e possibilitando um estoque de segurança menor. A política de revisão contínua (s, Q) - ponto de pedido, quantidade de pedido - determina que uma quantidade $Q$ é requisitada sempre quando o nível de estoque atinge um ponto de s unidades. Nesse modelo, a demanda e o tempo de entrega são variados, possibilitando decisões diferentes. Já na revisão periódica, as decisões sobre o ressuprimento são realizadas periodicamente, o que diminui o custo com o monitoramento do inventário e pode proporcionar uma programação das compras e do transporte e a racionalização dos recursos. Um modelo de política de revisão periódica é o (R, s, S) pedido de revisão, estoque de segurança, estoque máximo -, em que é avaliado o estoque atual para se decidir entre colocar ou não o pedido. Assim, a cada $\mathrm{R}$ unidades de tempo, os níveis de estoque são revisados. Caso a posição de estoque esteja igual ou inferior ao ponto de pedido s, uma ordem de 
reabastecimento é feita para se atingir $\mathrm{S}$ unidades estocadas. Mas se o inventário estiver acima do ponto s, no instante da revisão, não será realizado nenhum pedido.

Classificado o inventário, passou-se ao cálculo de estoque de segurança, lote de compra e ponto do pedido. Neste trabalho, para calcular o estoque de segurança, será utilizado o método com nível de atendimento, que fora empregado por Cauduro e Zucato (2011), e representa, segundo os autores, a parte da demanda que foi efetivamente atendida. Na presente pesquisa, considera-se uma variação do fator k de 0,8 até 1, de acordo com a classificação adotada, conforme Al-Qatawneh e Hafeez (2015). 0 cálculo do estoque segurança pelo método com nível de atendimento é representado pelas fórmulas:

$$
\begin{array}{ll}
\text { Es }=\sigma \times \mathrm{kx} \sqrt{\mathrm{LT}} & \text { se submetido à revisão contínua; } \\
\text { Es }=\sigma \times \mathrm{kx} \sqrt{\mathrm{LT}+\mathrm{R}} & \text { se submetido à revisão periódica; }
\end{array}
$$

em que:

Es = Estoque segurança em unidades;

$\sigma=$ desvio padrão em unidades;

$\mathrm{k}$ = fator de segurança;

$\mathrm{LT}=$ lead time;

$\mathrm{R}=$ período da revisão.

O ponto de pedido é expresso pela seguinte fórmula, de Pozo (2010):

Pp $=$ Es + C x LT

em que:

$\mathrm{Pp}=$ Ponto de pedido;

Es = Estoque de segurança;

$\mathrm{C}=$ Consumo;

$\mathrm{T}=$ Tempo para realizar o suprimento (lead time).

Ressalta-se que esta pesquisa foi cadastrada na Plataforma Brasil para submissão ao Comitê de Ética de Pesquisa da organização estudada, sendo aprovada por meio do Parecer Consubstanciado CEP no 2.372.973, de 09/11/2017, autorizando a realização da pesquisa na FHEMIG.

\section{Análise da situação-problema}

Seguindo o proposto por Mahagaonkar e Kelkar (2017) para elaboração da Curva ABC, inicialmente foram levantados os dados sobre quantitativos e preços unitários dos materiais médico-hospitalares consumidos no ano de 2017, na FHEMIG. Considerando apenas esse tipo de bem, foram elencados 1.423 itens de materiais, que apresentam diferentes códigos e especificações de tamanhos. Por exemplo, as luvas de procedimento são compradas em três tamanhos (pequeno, médio e grande), com e sem pó, originando seis itens e códigos de material. Assim, quando se desconsideram essas variações de especificação dentro de cada item, sendo contado apenas um item por material, reduz-se o inventário analisado para 333 tipos de item.

A seguir, foram calculados os gastos anuais por item, usando-se o Microsoft Excel. Ordenaram-se os valores encontrados em ordem decrescente e obteve-se o gasto acumulado. Com esses dados, foi possível estabelecer a classificação de cada material, conforme padrão citado na seção anterior. Após essa classificação, observa-se que 50 itens se enquadram no grupo A - 15\% do total - e compreendem $\mathrm{R} \$ 36.616 .187,53$; na classe B, tem-se 75 itens - 23\% da totalidade, que representam $\mathrm{R} \$ 7.022 .149,62$; e, por fim, na categoria C, estão 208 itens - 62\% do inventário -, com o valor de $\mathrm{R} \$ 2.331 .827,35$. 0 valor total gasto com material médico-hospitalar, no ano de 2017 , foi $\mathrm{R} \$ 45.970 .164,50$.

Com tais valores, foi possível construir a Figura 3, que demonstra a Curva ABC por meio do chamado gráfico de Pareto. Ressalta-se aqui que tal gráfico não demonstra todos os 333 itens, pela limitação imposta pelo tamanho da folha, mas permite uma avaliação da discrepância entre os custos totais, em que poucos bens têm valor financeiro alto e muitos materiais compreendem uma quantia bem menor.

\section{Figura 3 - Curva de Pareto}




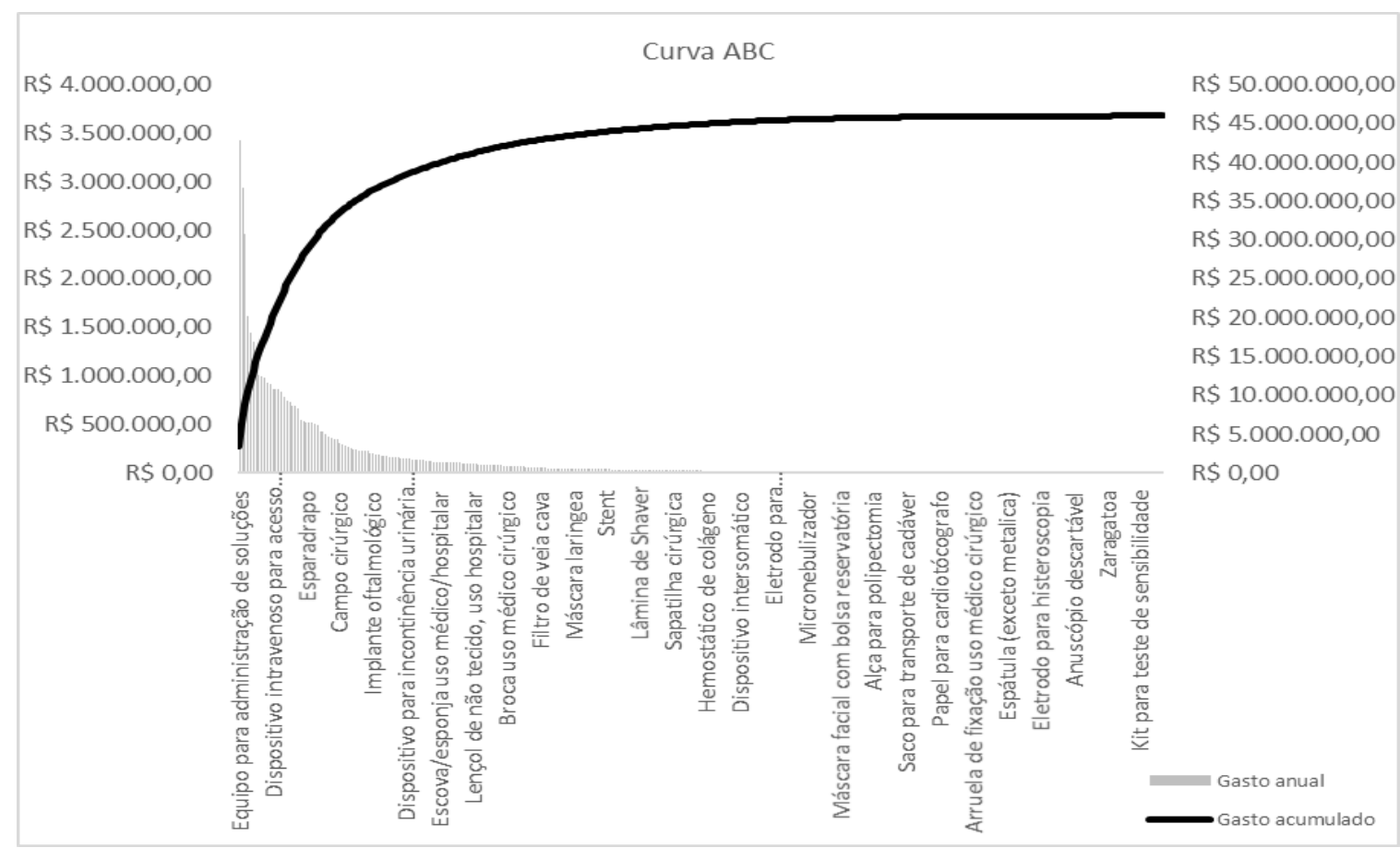

Fonte: Elaborado pelos autores

Utilizando-se os mesmos dados de 2017, foi elaborada a análise XYZ. Foram seguidos os passos descritos por Besta et al. (2015), sendo calculadas as médias, o desvio padrão e o coeficiente de cada material, por meio de uma planilha do Microsoft Excel. Ressalta-se que os cálculos foram realizados usando-se dados mensais. Encontrados os parâmetros estatísticos, passou-se à classificação dos itens nas três categorias da Curva XYZ, baseando-se no trabalho de Eleftheria (2017), destacado na seção anterior.

\section{Proposta de intervenção}

Após a definição dos nove subgrupos formados pela análise $\mathrm{ABC} / \mathrm{XYZ}$, passou-se ao cálculo do estoque de segurança, do ponto do pedido e do lote de compra. Para tanto, foram utilizados o fator de segurança $\mathrm{k}$ e as políticas de controle de estoque dos itens, de acordo com o subgrupo ao qual pertencem, consoante o estudo de Al-Qatawneh e Hafeez (2015). Quanto aos valores atribuídos ao índice $\mathrm{k}$, optou-se por utilizar os valores da tabela da distribuição normal, Z, atrelando-os ao nível de serviço desejado, seguindo o exemplo de Rosa, Xavier e Braga (2015). Assim, seguiu-se a análise de AlQatawneh e Hafeez (2015), que utilizaram nível de serviço e $\mathrm{k}$ a seguir: $100 \%$ de nível de serviço e $\mathrm{k}=$ 1 para as subcategorias AX, BX, CX, BY e CY; $90 \%$ de nível de serviço e $\mathrm{k}=0,9$ para os subgrupos AY e CY; e 80\% de nível de serviço e $\mathrm{k}=0,8$ para os itens de classificação AZ e BZ.

Assim, propõe-se que os itens $A X, B X, B Y, C X$ e CY tenham uma política de revisão contínua, e que $\mathrm{AY}, \mathrm{AZ}, \mathrm{BZ}$ e CZ tenham uma política de revisão periódica, resgatando os estudos de Stojanović e Regodić (2017). Quanto ao cálculo do estoque de segurança, foi utilizado o método de nível de atendimento, conforme Al-Qatawneh e Hafeez (2015), empregando-se as fórmulas 2 e 3 da seção anterior. No caso da organização estudada, o tempo de entrega é de 10 dias ou 0,33 mês. Utilizou-se o período de revisão de 2 meses, consoante Esmaili, Norman e Rajgopal (2015). O ponto de pedido foi calculado conforme a fórmula 4, apresentada na seção anterior, para todos os itens. Quando o nível de estoque atinge o ponto de pedido s, é solicitado um novo pedido ao fornecedor que assinou a respectiva ARP dos itens que serão solicitados. Assim, verifica-se a importância das atas para se manter o fornecimento com um tempo menor, influenciando a reposição dos estoques, com maior agilidade no reabastecimento.

Na política de revisão contínua - (s, Q), ponto de pedido, quantidade de pedido -, optou-se por um lote que compreende o consumo médio mensal de 4 meses, conforme trabalho de Costa e Guarnieri (2018). As autoras recomendam um lote que cobre 4 meses, considerando que pode haver falta de recursos financeiros por esse período, comprometendo o reabastecimento. Já na política de revisão periódica - $(\mathrm{R}, \mathrm{s}, \mathrm{S})$, pedido de revisão, estoque de segurança, estoque máximo -, o período de revisão $\mathrm{R}$ estimado é de 2 meses. Assim, a cada 2 meses, é verificado o nível de estoque dos materiais dos subgrupos AY, AZ, BZ e CZ. Caso o quantitativo seja igual ou inferior ao estoque de segurança calculado, será feito um novo pedido ao fornecedor da ARP vigente, em que o lote de compra será $Q=S-I$, em 
que I é o nível de estoque atual, ou seja, a aquisição solicitará uma quantidade para elevar o inventário ao estoque máximo do item. Considerando que pode haver atas de materiais que sigam as duas políticas de reposição de estoque com o mesmo fornecedor, recomenda-se que o período de revisão $\mathrm{R}$ coincida com a reposição da política contínua, quando for possível, para se evitarem múltiplas entregas do mesmo fornecedor. Os cálculos realizados de alguns materiais estão demonstrados na Tabela 1, para exemplificar os resultados alcançados.

Tabela 1 - Classificação ABC/XYZ, estoque de segurança, ponto de pedido e política de estoque

\begin{tabular}{|c|c|c|c|c|c|c|c|c|}
\hline Material & $\begin{array}{c}\text { Classificação } \\
\text { ABC }\end{array}$ & $\begin{array}{c}\text { Classificação } \\
\text { XYZ }\end{array}$ & $\mathrm{k}$ & $\begin{array}{l}\text { Estoque de } \\
\text { segurança }\end{array}$ & $\begin{array}{l}\text { Ponto do } \\
\text { pedido }\end{array}$ & $\begin{array}{l}\text { Tipo de } \\
\text { política }\end{array}$ & $\begin{array}{l}\text { Estoque } \\
\text { máximo }\end{array}$ & $\begin{array}{l}\text { Lote de } \\
\text { compra }\end{array}$ \\
\hline $\begin{array}{l}\text { Equipo para } \\
\text { administração } \\
\text { de soluções }\end{array}$ & A & $\mathrm{X}$ & 3,9 & 11.275 & 29.883 & s, Q & 236.830 & 225.555 \\
\hline $\begin{array}{l}\text { Avental para } \\
\text { uso médico/ } \\
\text { odontológico }\end{array}$ & A & Z & 0,84 & 8.037 & 10.515 & $R, s, S$ & 38.075 & 27.560 \\
\hline $\begin{array}{l}\text { Frasco para } \\
\text { administração } \\
\text { de nutrição }\end{array}$ & B & $\mathrm{X}$ & 3,9 & 7.176 & 11.653 & s, Q & 61.436 & 54.259 \\
\hline $\begin{array}{l}\text { Grampeador } \\
\text { cirúrgico }\end{array}$ & B & Y & 1,28 & 7 & 9 & s, Q & 29 & 22 \\
\hline $\begin{array}{l}\text { Agulha de } \\
\text { punção }\end{array}$ & $\mathrm{C}$ & $\mathrm{X}$ & 3,9 & 61 & 92 & s, Q & 433 & 371 \\
\hline $\begin{array}{l}\text { Isolador de } \\
\text { condutor de } \\
\text { pressão para } \\
\text { hemodiálise }\end{array}$ & $\mathrm{C}$ & Z & 0,84 & 135 & 214 & $R, s, S$ & 1.086 & 872 \\
\hline
\end{tabular}

Fonte: Elaborado pelos autores

Foram classificados 102 itens no grupo X, 89 na classe Y e 142 na Z. Conforme observado na Tabela 1, unidas as duas Curvas, é possível verificar que existem itens AZ caros e com demanda irregular, como o avental para uso médico/odontológico, e itens CX baratos e com utilização contínua, como agulhas de punção. Tais constatações refletem a importância de se considerarem outros critérios, além do financeiro, como defendem Gonçalves et al. (2015) e Clevert et al. (2007).

Para estimar as possíveis economias de recursos financeiros, com a utilização das políticas contínua e periódica propostas - s, Q e R, s, S, respectivamente -, foi realizada uma projeção da redução de custos. Considerando dois pedidos durante o ano, apenas para os itens de revisão periódica (AY, AZ, BZ, CZ), que possuem demanda mais irregular, foi observada uma redução inicial de $R \$ 3.900 .006,43$, que correspondem a 8,6\% do gasto anual. Tal estimativa corrobora a ideia de que a adoção das práticas sugeridas por este trabalho pode permitir um uso mais racional do orçamento destinado às compras durante o ano.

\section{Conclusões e contribuições tecnológicas e sociais}

Diante das peculiaridades da gestão de materiais na saúde pública, o objetivo geral deste trabalho foi propor políticas de gestão de materiais médico-hospitalares, por meio da análise $\mathrm{ABC} / \mathrm{XYZ}$, que permitiram estabelecer uma classificação baseada em valor e demanda e políticas de gestão de estoques. Foram elaboradas, inicialmente, as classificações ABC e XYZ, separadamente. Em seguida, ambas foram combinadas. Ao se cruzarem essas duas vertentes - custo e demanda -, fica visível a importância de se considerarem outras variáveis, além da financeira, conforme defendido por Clevert et al. (2007), Gonçalves et al. (2015) e Mahagaonkar e Kelkar (2017), pois ao considerar a variação da demanda, com a utilização da curva XYZ, os materiais mais baratos e com utilização constante não são menosprezados. Verifica-se, por um lado, que há itens caros e usados em casos mais esporádicos - AZ, 
que devem ser estocados em quantidade moderada. Por outro lado, tem-se materiais com custo menor e que são utilizados com mais regularidade ao longo do ano - CX -, os quais não receberiam a devida atenção caso se analisasse apenas a curva $\mathrm{ABC}$, podendo gerar faltas ou excessos.

Outro ponto relevante da classificação dos itens foi permitir a criação de subgrupos com características semelhantes, que admitem a aplicação de políticas de revisão de estoque mais adequadas. Tal categorização é relevante diante da grande variedade de itens no estoque de materiais médico-hospitalares de uma rede hospitalar, consoante a visão de Duarte et al. (2015), Figueiredo e Aquino (2016), Gonçalves et al. (2015), Santos et al. (2012). Diante das particularidades da gestão de materiais, que se mostra mais complexa em ambientes hospitalares, conforme aponta Hafnika (2016) e Souza e Rosa (2015), a divisão dos itens em classes também facilita uma gestão mais otimizada. A partir das nove subclasses, foram adotadas a revisão contínua para os materiais mais caros e/ou com maior regularidade do uso (AX, BX, BY, CX e CY) e a revisão periódica para os itens com menor regularidade de consumo ( $\mathrm{AY}, \mathrm{AZ}, \mathrm{BZ}$ e $\mathrm{CZ}$ ). Assim, os primeiros contam com um acompanhamento continuado, para se evitar a falta, visto que têm demanda mais frequente, e impedir excessos e perdas, pelo maior valor financeiro envolvido. Já os materiais de revisão periódica, por terem consumo mais esporádico, têm seu monitoramento realizado em determinados momentos.

Após a divisão dos itens, conforme as políticas de revisão de estoque, foram calculados estoque de segurança, ponto de pedido e lote de compra. Quanto ao estoque de segurança, no caso dos materiais submetidos à política contínua, foi considerado o lead time. Para os itens de política periódica, foram utilizados o tempo de entrega e o tempo de revisão, para adequar o nível do estoque mínimo a esses indicadores, que também o influenciam. Assim, percebe-se que o ES foi um pouco superior no caso dos itens de revisão periódica, pois considerou o consumo do período entre as revisões. Assim, conclui-se que a classificação $\mathrm{ABC}-\mathrm{XYZ}$ permite a adoção de políticas de revisão de estoque que possibilitam controles mais adequados ao custo e à rotatividade do inventário, dada a variabilidade deste, refletindo nos estoques de segurança, ponto do pedido e lote de compra mais apropriados, para se evitarem excesso, faltas e perdas, conforme a visão de Póvoa e Chaves (2014), Santana e Piper (2017) e Stojanović e Regodić (2017). Tais autores consideram que avaliar as variações da demanda no planejamento de compras apresenta mais racionalidade e eficiência no uso dos recursos financeiros e nos estoques mantidos pela organização.

Reitera-se a relevância das ARP, que possibilitam a utilização das rotinas de aquisição aqui propostas, pelo fato de reduzirem o tempo para compras dos órgãos e entidades públicos. O SRP é recomendado pela legislação e pela literatura, para estabelecer uma possibilidade de aquisição de materiais, de acordo com as necessidades da organização, pelo prazo determinado. Destacam-se as economias de recursos financeiros que são obtidas, considerando um quantitativo menor em estoque e mais otimizado com as reais demandas. Isso evita excessos e possíveis perdas, além de exigir menos espaço físico e custos menores de manutenção dos inventários e permitir planejar os pedidos nos momentos em que há mais verbas disponíveis para empenho, ao longo do ano.

Como limitações da pesquisa, em relação ao tempo de entrega, ressalta-se que o lead time foi considerado fixo, sem levar em conta possíveis oscilações que podem ocorrer (greves, atrasos dos fabricantes, atrasos nos pagamentos por falta de recursos financeiros, entre outras). Também não foram considerados os períodos entre a solicitação e a liberação de empenhos (recursos financeiros) para as unidades realizarem uma nova aquisição, no SRP. Considerando que se trata de pesquisa documental, pode haver itens em que o quantitativo documentado informado não corresponda ao estoque real da organização estudada. Assim, recomenda-se coletar dados dessas variações e empregálos nos cálculos referentes ao estoque. Por fim, sugere-se replicar as políticas propostas no presente trabalho aos demais materiais utilizados na organização.

\section{Referências}

Al-Qatawneh, L., \& Hafeez, K. (2015). Critical-to-life classification for managing inventory in a healthcare supply chain. International Journal of Intelligent Enterprise, 3(1), 54-78. Recuperado de https://doi.org/10.1504/IJIE.2015.07349121

Alemão, M. M., Gonçalves, M. A., \& Drumond, H. A. (2013). Estudo da utilização da informação de custos como ferramenta de gestão em organização pública: o estudo do SIGH-custos. Perspectivas em Gestão \& Conhecimento, 3(1), 210-226. Recuperado de http://periodicos.ufpb.br/ojs2/index.php/pgc.03

Almeida, A. V. C., Tavares, D. M. L., Melo, A. C. S., Nunes, D. R. L., \& Negrão, L. L. L. (2016). Melhorias no ressuprimento de itens de consumo em uma universidade pública no Estado do Pará. Revista Perspectivas Online: exatas \& engenharia, 6(16), 12-26. Recuperado de http:// www.seer.perspectivasonline.com.br/index.php/exatas_e_engenharia/article/view/66029

Alvear, F. T., \& Mello, R. B. (2016). Importância da tecnologia da informação (TI) para a gestão de estoques em uma farmácia hospitalar. Revista Interação, 18(1), 91-108. Recuperado de http:// 
interacao.newwp.unis.edu.br/wp-content/uploads/sites/80/2016/ 08/INTERA\%C3\%87\%C3\%830vol-18-ARQUIVO-\%C3\%9ANICO-18-07.pdf 15

Araújo, E. A., Araújo, A. D., \& Musetti, M. A. (2012). Estágios organizacionais da logística: estudo de caso em organização hospitalar filantrópica. Revista Produção, 22(3), 549-563. Recuperado de http:// dx.doi.org/10.1590/S0103-65132012005000 04523

Besta, P., Janovská, K., \& Bumbová, M. (2015). The possibilities of the structure and variability evaluation of inventory consumption. International Scientific Journal about Logistics, 2(3), 21-25. doi:10.22306/al.v2i3.4406

Campos, F., \& Maria, T. C. (2015). Aspectos críticos do instituto da estabilidade do servidor público e o princípio da eficiência na administração pública. Revista da $A M D E, 13,38-60$. Recuperado de http:// revista.amde.org.br/index.php/ramde/article/view/266/pdf

Cauduro, V. D., \& Zucatto, L. C. (2011). Proposição de lote econômico como estratégia de compra para farmácia hospitalar municipal. Contexto, 11(20), 73-84. Recuperado de http://seer.ufrgs.br/ index.php/ConTexto/article/view/2228019

Clevert, D. A., Stickel, M., Jung, E. M., Reiser, M., \& Rupp, N. (2007). Cost analysis in interventional radiology - A tool to optimize management costs. European Journal of Radiology, 61(1), 144-149. Recuperado de https://doi.org/10.1016/j.ejrad.2006.08.01104

Costa, C. D. F., \& Guarnieri, P. (2018). Gestão da aquisição e dos estoques de medicamentos: estudo de caso no Hospital Universitário de Brasília (HUB). Revista Foco, 11(2), 28-52. Recuperado de https:// doi.org/10.28950/1981-223x_revistafocoadm/2018.v11i2.52427

Decreto n. 7.892, de 23 de janeiro de 2013. (2013). Regulamenta o Sistema de Registro de Preços previsto no art. 15 da Lei no 8.666, de 21 de junho de 1993. Brasília, DF. Recuperado de http:// www.planalto.gov.br/ccivil_03/_ato2011-2014/2013/decreto/d7892.htm

Duarte, N. C. M., Bitar, J. P. S, Miglioli, J. P., Pereira, M. M. S., Yodono, N. B. P., Araújo, T. R., \& Pazin-Filho, A. (2015). Gestão de compras em um hospital de ensino terciário: um estudo de caso. Revista Medicina Ribeirão Preto, 48(1), 48-56. Recuperado de http://dx.doi.org/10.11606/ issn.2176-7262.v48i1p48-5625

Eleftheria, M. (2017). Kanban System Design for Hospital Pharmacy - Case study. Journal of Statistical Science and Application, 5(1-2), 30-38. Recuperado de https://10.17265/2328-224X/2017.0102. 00304

Esmaili, N., Norman, B. A., \& Rajgopal, J. (2015). A Heuristic for Selecting Multi-item Inventory Review Policies. Proceedings of 65th Industrial and Systems Engineering Research Conference. Recuperado de https://www.researchgate.net/publication/319018597_A_Heuristic_for_Selecting_Multiitem_Inventory_Review_Policies

Ferreira, R.; Luz, J.; Amorim, J. (2016). Proposta de melhorias na administração de materiais para a empresa Rocco Restaurante Grill Contemporâneo. Caderno Científico - CECIESA GESTÃO, 2(1), 57-65. Recuperado de http://siaiap32.univali.br/seer/ index.php/cccg/article/view/10204/5744

Figueiredo, W. B., \& Aquino, S. (2016). Modelo de aquisição de insumos hospitalares para prevenção de infecção nosocomial em uma unidade de terapia intensiva baseada no sistema ABC e XYZ. Internacional Journal of Health Management Review, 2(1), 84-107. Recuperado de http://doi 10.21902/jhmreview.v2i1.34127

Fuccia, I. R., Matsufugi, M. T., Martins, M. C., Sforsin, A. C. P., \& Pinto, V. B. (2017). Estudo de viabilização e proposta de implantação de sistema kanban em uma central de atendimento por dose individualizada. Revista Administração em Saúde, 17(67), 1-28. Recuperado de http://dx.doi.org/ 10.23973/ras.67.26 201715

Gonçalves, M. C., Gonçalves, T. B. L., Tavares, D. M. L., Melo, A. C. S., \& Nunes, D. R. T. (2015). Sistema para geração automática de requisição de compras e controle de atas de registro de preços: um estudo de caso no setor elétrico. Revista Gestão Industrial, 11(4), 134-153. Recuperado de https:// revistas.butfpr.edu.br/revistagi/article/view/2886/247306

Günergören, H., \& Dağdeviren, O. (2017). An Excel-Based Inventory Control System Based on ABC and VED Analyses for Pharmacy: A Case Study. Galore International Journal of Health Sciences and Research, 2(1), 11-17. Recuperado de http://www.gijhsr.com/GIJHSR_Vol.2_Issue.1_March2017/ 3.pdf24

Hafnika, F., Farmaciawaty, D. A., Adhiutam, A., \& Basri, M. H. (2016). Improvement of Inventory Control Using Continuous Review. The Asian Journal of Technology Management, 9(2), 109-119. Recuperado de http://dx.doi.org/10.12695/ajtm.2016. 9.2.527

Mahagaonkar, S. S., \& Kelkar, A. A. (2017). Application of ABC Analysis for Material Management of a Residential Building. International Research Journal of Engineering and Technology, 4(8), 614-620. Recuperado de https://irjet.net/archives/V4/i8/IRJET-V4I8108.pdf22

Marconi, M. A., \& Lakatos, E. M. (2010). Fundamentos de metodologia científica (7. ed.). São Paulo: Atlas. 
Meaulo, M. P., Pensutti, M. (2011, dezembro). A gestão de estoques em ambientes hospitalares: analisando a utilização de ferramentas de gestão de materiais em ambientes hospitalares públicos e filantrópicos no município de Santa Bárbara D’Oeste. Anais do 8o Congresso Virtual Brasileiro de Administração. Recuperado de http://www.convibra.com.br/ upload/paper/adm/adm_3253.pdf

Melo, A. B., Gomes, B. R. S., Pinheiro, B. S. B., Martins, L. F. J., Palheta, M. G., Santos, R. S. U., \& Silva, I. M. (2016). A gestão de materiais médico-hospitalar em hospital público. Revista Eletrônica Gestão \& Saúde, 7(1), 369-387. Recuperado de http://dx.doi.org/10.18673/gs.v7i1.22086 27

Minas Gerais. (2021). Portal da Transparência. Despesas da Fundação Hospitalar de Minas Gerais no ano de 2019. Recuperado de https://www.transparencia.mg.gov.br/despesa-estado/despesa/ despesa-orgaos/2019/01-01-2019/31-12-2019/3909/1869/469/20/42

Póvoa, B. B., \& Chaves, G. L. D. (2014). Ponto de ressuprimento para materiais com demanda sazonal e aleatória: o caso de uma empresa de grande porte. Revista Gestão Industrial, 10(3), 584-618. https:// 10.3895/gi.v10i3.185323

Pozo, H. (2010). Administração de Recursos Materiais e Patrimoniais: uma abordagem logística (5. ed.). São Paulo: Atlas.

Raimundo, E. A., Dias, C. N., \& Guerra, M. (2015). Logística de medicamentos e materiais em um hospital público do Distrito Federal. Revista de Administração Hospitalar e Inovação em Saúde, 12(2), 61-69. Recuperado de http://dx.doi.org/10. 21450/rahis.v12i2.238423

Rosa, R. C., Xavier, R. O., \& Braga, L. B. M. (2015). Otimização dos processos de controle de estoque de materiais e medicamentos em uma farmácia hospitalar. Revista Petra, 1(2), 261-275. Recuperado de http://dx.doi.org/10.15601/2359-5302/ptr.v1n2p261-7509

Santana, M. L., \& Piper, H. (2017). Proposta de melhoria de layout e a curva ABC no setor de estoque: um estudo de caso. Revista Ciência (In) Cena, 1(5), 30-50. Recuperado de http:// periodicos.estacio.br/index.php/cienciaincenabahia/article/viewFile/3241/pdf324111

Santos, P. S. A., Fank, O. L., \& Varela, P. S. (2012). Determinantes do nível dos estoques públicos: mensuração nas capitais dos estados da região sul do Brasil. Revista Eletrônica Estratégia \& Negócios, 5(2), 28-55. Recuperado de http://portaldeperiodicos.unisul.br/index.php/EeN/article/view/575

Santos, M. Q. A., Scheidegger, A. P. G., Favaretto, F., \& Lima, R. S. (2014, outubro). Gestão de estoques de materiais de consumo na prefeitura do campus da Universidade Federal de Itajubá. Anais do Encontro Nacional de Engenharia de Produção, Curitiba, PR, Brasil, 34. Recuperado de https:// www.researchgate.net/publication/317291027

Souza, D. O., \& Rosa, R. S. (2015). Compras nacionais e internacionais em um hospital de capacidade extra. In R. Bordin, D. J. Demarco, \& F. B. Meira. Gestão em saúde no Rio Grande do Sul: casos, análises e práticas. (Cap. 2. v. 2. pp. 253-279). Porto Alegre: Evangraf. Recuperado de http://hdl.handle.net/ $10183 / 159760$

Souza, M. R., Cunha, M. H. B., \& Leite, C. E. (2016). O processo de gestão de estoques no setor de transporte público: um estudo de caso. Universitas Gestão e TI, 6(2), 67-76. Recuperado de 0.5102/ un.gti.v6i2.3886

Stojanović, M., \& Regodić, D. (2017). The Significance of the Integrated Multicriteria ABC-XYZ Method for the Inventory Management Process. Acta Polytechnica Hungarica, 4(5), 29-48. Recuperado de https://10.12700/APH.14.5.2017.5.314

Tridapalli, J. P., Fernandes, E., \& Machado, W. V. (2011). Gestão da cadeia de suprimento do setor público: uma alternativa para controle de gastos correntes no Brasil. Revista de Administração Pública, 45(2), 401-433. Recuperado de http://www.scielo.br/pdf/rap/v45n2/06.pdf20. 